Journal of Autism and Developmental Disorders, Vol. 26, No. 2, 1996

\title{
Brief Report: Comorbid Psychiatric Disorders of Autistic Disorder
}

\author{
Luke Y. Tsai ${ }^{1}$ \\ Developmental Disorders Clinic, University of Michigan Medical Center
}

There are several medical conditions that have been recognized with relatively high frequency in individuals with Autistic Disorder (AD). About $30 \%$ of people with $\mathrm{AD}$ also have seizure disorders; $2-5 \%$ have fragile $\mathrm{X}$ syndrome; and 1-3\% have tuberous sclerosis. In addition to these coexisting medical conditions, many autistic individuals also develop other behavioral and/or psychiatric symptoms in addition to the core autistic symptomatologies (i.e., impairment in social interaction, impairment in communication, and restricted, repetitive and stereotyped patterns of behavior, interests, and activities), that may be considered clinical manifestations of comorbid psychiatric disorders. The additional behavioral and/or psychiatric symptoms have been described by many investigators. Rutter, Greenfield, and Lockyer (1967) reported that among the 63 children with Infantile Psychosis, $48 \%$ were hyperkinetic at the time of first hospitalization, $43 \%$ had morbid preoccupation, $37 \%$ had obsessive phenomena, $70 \%$ had stereotyped mannerism, $25 \%$ had self-injury, and $60 \%$ had anxiety or fear. Simons (1974) reported that compulsive behavior was observed in every child with a clear-cut diagnosis of autism. Ando and Yoshimura (1979) reported that among the 47 autistic children (age range 6-14 years), 36\% had hyperactivity; $68 \%$ had stereotyped behavior; $43 \%$ had self-injury; and $17 \%$ had fear. In a follow-up study of autistic adult males, Rumsey, Rapoport, and Sceery (1985) reported that $86 \%$ of these males continued to demonstrate stereotyped, compulsive behaviors, including arranging objects and other objects, and phonic tics. In a study of reliability and diagnostic validity of the Autism Diagnostic Interview (ADI) in 16 autistic subjects (mean age $=13.26$ years, $S D=3.38)$, Le Couteur et al. (1989) reported that $73 \%$ of

${ }^{1}$ Address all correspondence to Luke Y. Tsai, Developmental Disorders Clinic, University of Michigan Medical Center, 1500 E. Medical Center Drive, Ann Arbor, Michigan 48109-0390. 
the subjects had separation anxiety, $89 \%$ had stereotyped utterances, $88 \%$ had unusual preoccupations, $55 \%$ demonstrated verbal rituals, $81 \%$ showed compulsions/rituals, $69 \%$ had hand-finger mannerism, and $63 \%$ had unusual sensory interests. Fombonne (1992) also studied ADI in 20 French autistic subjects (age range 11-26 years). He reported that $74 \%$ of the subjects had separation anxiety, $50 \%$ had stereotyped utterances, $53 \%$ had unusual preoccupations, $16 \%$ showed compulsions or rituals, $74 \%$ had hand-finger mannerism, and $42 \%$ had unusual sensory interests. In a follow-up study of 66 autistic subjects (86\% were below 12 years of age on follow-up) in Hong Kong, Chung, Luk, and Lee (1990) noted that $47 \%$ of these children were hyperactive, $64 \%$ had poor attention and concentration, $24 \%$ had self-injurious behaviors, $23 \%$ had fear or phobia, $9 \%$ had depressive mood, $44 \%$ had irritability or agitation, $29 \%$ showed inappropriate affect, $11 \%$ had sleep problem, and $8 \%$ exhibited tics. These investigators, however, did not specifically investigate the incidence of diagnosable psychiatric disorders based on any commonly used diagnostic criteria in their samples.

Based on the observations as described above, the DSM-III, DSM-III$R$, and DSM-IV diagnostic classification systems have viewed the relatively high frequency, of motor stereotypies (e.g., hand-clapping, peculiar hand movements, rocking, dipping and swaying movements of the whole body) and verbal stereotypies (e.g., repetition of words or phrases) as diagnostic criteria items of $\mathrm{AD}$ (i.e., under the categories of qualitative impairments in communication category, and markedly restricted repertoire of activities and interests, respectively). However, these motor and verbal stereotypies are noted frequently in individuals with Tourette syndrome (TD) and these motor and verbal symptoms have been used as diagnostic features of TD. On the other hand, the DSM classifications considered the other behavioral and/or psychiatric symptoms as "associated features" of $\mathrm{AD}$ (e.g., abnormalities of posture and motor behavior: arm-flapping, jumping, grimacing; odd responses to sensory input or being fascinated by some sensations: smelling and/or touching people or objects; self-injurious behavior: headbanging, or finger-, hand-, or wrist-biting; abnormalities or mood: excessive fearfulness in response to harmless objects or events; generalized anxiety and tension; abnormalities of mood: labile mood, giggling or weeping for no apparent reason, apparent absence of emotional reactions; abnormalities in sleeping: recurrent awakening at night with rocking).

\section{NEW DIAGNOSTIC CONCEPT OF COMORBID PSYCHIATRIC DISORDERS}

Research on the specific relationship between these associated features and the core autistic symptoms is sparse, if any. It is not clear whether these 
emergent behavioral and/or psychiatric symptoms are developmentally related symptoms/behaviors of $A D$ or if these symptoms should really be considered as "comorbid psychiatric disorders." However, there is an accumulation of case reports describing specific types of psychiatric disorders occurring in individuals with $\mathrm{AD}$. These include reports of unipolar and bipolar affective disorders (Ghaziuddin \& Tsai, 1991; Gillberg, 1985; Komoto, Usui, \& Hirata, 1984; Steingard \& Biederman, 1987; Lainhart \& Folstein, 1994), obsessive-compulsive disorder (Rutter et al., 1985; Tsai, 1992; McDougle, Price, \& Goodman, 1990), schizophrenia (Clarke, Littlejohns, Gorbett, \& Joseph, 1988; Petty, Ornitz, Michelman, \& Zimmerman, 1984; Volkmar \& Cohen, 1991), Tourette syndrome (Barabas \& Matthews, 1983; Comings \& Comings, 1991; Realmuto \& Main, 1982; Sverd, Montero, \& Gurevich, 1993). It is conceivable that some of the associated features may be the diagnostic features of other coexisting psychiatric disorders. The question then would be: How frequently do these comorbid psychiatric disorders exist?

Many experienced clinicians in the field of autism have shared their observations that when these additional behavioral and/or psychiatric symptoms do exist in some autistic individuals, only the higher functioning autistic persons, a minority of autistic population, were more likely to receive additional DSM psychiatric diagnoses. Clinicians are usually reluctant to make additional psychiatric diagnosis in the lower functioning and/or nonverbal autistic individuals who are unable to or incapable of providing diagnostic information via diagnostic interviewing or patient self-report scales (Tsai, 1994). The current problem, the lack of a reliable and valid alternative diagnostic method to be applied to the lower-functioning and/or nonverbal autistic population, is another contributory factor.

To render more effective treatment to people with $A D$, the current assessment technology must be advanced and refined. To accomplish this goal, it may require some modifications of the contemporary diagnostic criteria of certain psychiatric disorders when dealing with an autistic population. For example, the diagnosis of Obsessive-Compulsive Disorder may be considered in lower functioning autistic individuals even in the absence of clear ego-dystonicity, or the diagnosis of Major Depression may be considered in nonverbal and/or lower functioning autistic persons even in the absence of subjectively reported depressed mood, worry, guilty feeling, and suicidal ideation. Attention Deficit Hyperactivity Disorder should be added as an additional diagnosis in autistic individuals with severe or profound mental retardation (Ghaziuddin, Tsai, \& Alessi, 1992).

\section{CONCLUSION}

The behavioral and/or psychiatric symptoms in autistic people as described above have been viewed by many professionals dealing with autistic 
populations as "associated features of autism" that may result from these individuals' inability to cope with the environmental demands and physical discomfort. Traditionally, in treating individuals with autism, special education intervention including behavioral modifications has been the main emphasis. Such an approach has made some progress in milder and uncomplicated cases of autism. However, if many of these behavioral and/or psychiatric symptoms in those with more severe associated features can be viewed as symptoms of various comorbid psychiatric disorders, there are data suggesting that with an appropriate evaluation, predrug workups, a specific diagnosis, and multiple measures of outcome, pharmacotherapy can be a safe and efficacious adjunct treatment for some symptoms in autistic persons. Nevertheless, the data presented here were obtained mainly from autistic children. A great deal of work remains to be done Future research should put more emphasis on developing agreeable, reliable, and valid diagnostic instruments for identifying comorbid psychiatric disorders in autistic people. Future research should also emphasize employing a randomized double-blind placebo-controlled crossover design, as well as involving multicenters and using uniformed diagnostic criteria to study autistic adolescents and adults.

\section{REFERENCES}

Ando, H., \& Yoshimura, I. (1979). Effects of age on communication skill levels and prevalence of maladaptive behaviors in autistic and mentally retarded children. Journal of Autism and Development Disorders, 9, 83-93.

Barabas, G., \& Matthews, W. S. (1983). Coincident infantile autism and Tourette syndrome: A case report. Joumal of Autism and Developmental Disorders, 13, 280-281.

Chung, S. Y., Luk, S. L., \& Lee, P. W. H. (1990). A follow-up study of Infantile Autism in Hong Kong. Journal of Autism and Developmental Disorders, 20, 221-232.

Clarke, D. J., Littlejohns, C. S., Corbett, J. A., \& Joseph, S. (1989). Pervasive developmental disorders and psychosis in adult life. British Joumal of Psychiatry, 155, 692-699.

Comings, D. E., \& Comings, B. G. (1991). Clinical and genetic relationship between autism-pervasive developmental disorder and Tourette syndrome: A study of 19 cases. American Journal of Medical Genetics, 39, 180-191.

Fombonne, E. (1992). Diagnostic assessment in a sample of autistic and developmentally impaired adolescents. Journal of Autism and Developmental Disorders, 22, 563-581.

Ghaziuddin, M., \& Tsai, L. (1991). Depression in autistic disorder. British Joumal of Psychiatry, $159,721-723$.

Ghaziuddin, M., Tsai, L., \& Alessi, N. (1992). ADHD and PDD. Joumal of the American Academy of Child and Adolescent Psychiatry, 31, 567.

Gillberg, C. (1985). Asperger's syndrome and recurrent psychosis-A case study. Journal of Autism and Developmental Disorders, 15, 389-397.

Komoto, J., Usui, S., \& Hirata, J. (1984). Infantile autism and affective disorder. Joumal of Autism and Developmental Disorders, 14, 81-84.

Lainhart, J. E., \& Folstein, S. E. (1994). Affective Disorders in people with Autism: A review of published cases. Journal of Autism and Developmental Disorders, 24, 587-601. 
Le Couteur, A., Rutter, M., Lord, C., Rios, P., Robertson, S., Holdgrafer, M., \& McLennan, J. (1989). Autism Diagnostic Interview: A standardized investigator-based instrument. Journal of Autism and Developmental Disorders, 19, 363-387.

McDougle, C. J., Price, L. H., \& Goodman, W. K. (1990). Fluvoxamine treatment of coincident Autistic Disorder and Obsessive-Compulsive Disorder: A case report. Joumal of Autism and Developmental Disorders, 20, 537-543.

Petty, L. K. Ornitz, E. M., Michelman, J. D., \& Zimmerman, E. G. (1984). Autistic children who become schizophrenic. Archives of General Psychiatry, 41, 129-135.

Realmuto, G. M., \& Main, B. (1982). Coincidence of Tourett's disorder and infantile autism. Journal of Autism and Developmental Disorders, 12, 367-372.

Rumsey, J. M., Rapoport, J. L., \& Sceery, W. R. (1985). Autistic children as adults: Psychiatric, social, and behavioral outcomes. Joumal of the American Academy of Child Psychiatry, 24, 465-473.

Rutter, M., Greenfeld, D., \& Lockyer, L. (1967). A five to fifteen year follow-up study of Infantile Psychosis: II. Social and behavioral outcome. British Journal of Psychiatry, 113, $1183-1199$.

Simons, J. M. (1974). Observations on compulsive behavior in autism. Joumal of Autism and Childhood Schizophrenia, 4, 1-10.

Steingard, R., \& Biederman, J. (1987). Lithium responsive manic-like symptoms in two individuals with autism and mental retardation. Journal of American Academy of Child Adolescent Psychiatry, 26, 932-935.

Sverd, J., Montero, G., \& Gurevich, N. (1993). Cases for an association between Tourette syndrome, Autistic Disorder, and Schizophrenia-Like Disorder. Journal of Autism and Developmental Disorder, 23, 407-413.

Tsai, L. (1992). Diagnostic issues in high-functioning autism. In E. Schopler \& G. B. Mesibov (Eds.), High-functioning individuals with autism (pp. 11-40). New York: Plenum Press.

Tsai, L. (1994). Comorbid disorders of autistic disorder. Presented at the Special Interest Group of Annual Meeting of American Academy of Child and Adolescent Psychiatry, New York City.

Volkmar, F. R., \& Cohen, D. J. (1991). Comorbid association of autism and schizophrenia. American Joumal of Psychiatry, 148, 1705-1707. 